\title{
Supporting Everyday Function in Chronic Pain Using Wearable Technology
}

\author{
Aneesha Singh \\ UCL Interaction Centre \\ UCL, London, UK \\ aneesha.singh@ucl.ac.uk
}

\author{
Nadia Bianchi-Berthouze \\ UCL Interaction Centre \\ UCL, London, UK \\ n.berthouze@ucl.ac.uk
}

\author{
Amanda CdeC Williams \\ Dept. of Clinical, Educational \& Health \\ Psychology, UCL, London, UK \\ amanda.williams@ucl.ac.uk
}

\begin{abstract}
While most rehabilitation technologies target situated exercise sessions and associated performance metrics, physiotherapists recommend physical activities that are integrated with everyday functioning. We conducted a 1-2 week home study to explore how people with chronic pain use wearable technology that senses and sonifies movement (i.e., movement mapped to sound in real-time) to do functional activity (e.g., loading the dishwasher). Our results show that real-time movement sonification led to an increased sense of control during challenging everyday tasks. Sonification calibrated to functional activity facilitated application of pain management techniques such as pacing. When calibrated to individual psychological needs, sonification enabled serendipitous discovery of physical capabilities otherwise obscured by a focus on pain or a dysfunctional proprioceptive system. A physiotherapist was invited to comment on the implications of our findings. We conclude by discussing opportunities provided by wearable sensing technology to enable better functioning, the ultimate goal of physical rehabilitation.
\end{abstract}

\section{Author Keywords}

Everyday function; wearables; chronic pain; sonification; feedback; home rehabilitation; ubiquitous technology.

\section{ACM Classification Keywords}

H.5.m. Information interfaces and presentation (e.g., HCI): Miscellaneous;

\section{INTRODUCTION}

Movement sensing technology for physical rehabilitation is increasingly being used in home environments either to support exercise or to quantify activity. More recently realtime feedback has proved to be helpful in supporting physical rehabilitation $[33,42]$ and studies have suggested its use in facilitating functional activity [7,41]. However, most physical rehabilitation technology is designed to target

This work is licensed under a Creative Commons Attribution International 4.0 License.

Copyright is held by the owner/author(s). CHI 2017, May 06-11, 2017, Denver, CO, USA ACM 978-1-4503-4655-9/17/05.

http://dx.doi.org/10.1145/3025453.3025947 exercise rather than functional activity. Here, we use the term exercise to refer to prescribed or structured exercises and stretches, whilst the term functional activity encompasses activities of daily living such as housework and socialising. In this paper, we investigate how movement sensing and feedback technology can facilitate functional activity in people with chronic pain (CP).

To this end, we conducted a 1-2 week in-the-wild home study using a wearable prototype to support the daily activities of people with CP. The prototype, based on [42], tracked the movement of the part of the body where it was worn and transformed the movement into real-time sound feedback. While the study reported in [42] aimed to investigate how the device could support in-situ exercise, participants felt that the device could also be useful for functional activity, which is important in CP rehabilitation. We also report findings from a post-study interview with a physiotherapist specialising in CP management, cued by the participants' analysed data, videos and diaries.

This paper makes three contributions. First, we show that real-time movement sonification can facilitate and support self-managed functional activity in people's homes. Second, we identify strategies people used to calibrate the sonification, to obtain information needed to build confidence in functional activities. Finally, we discuss opportunities provided by wearable sensing technologies and how they should be designed to enable functioning, the main goal of rehabilitation in CP.

CHRONIC PAIN AND SELF-MANAGEMENT OF ACTIVITY Chronic pain (CP) is a long-term illness with no cure. Despite being a debilitating condition that affects $19 \%$ of the population [13], it is usually self-managed with very little input from health professionals. CP can be maladaptive as it is not always in response to an injury but due to over-sensitisation of the nervous system, which causes spontaneous creation or amplification of pain signals [19]. Regular physical activity can reduce physical weakening and stiffness [19] but people with CP can struggle to do physical activity due to pain and related emotional factors [44] such as fear of injury or of increasing pain. These factors can cause people to adopt behaviours that further restrict movement, exacerbate pain and limit engagement in everyday functional activity. People with $\mathrm{CP}$ are often unaware of these habitual protective behaviours [46] and need external support to overcome them. 
Pain management programmes (generally one month-long) aim to provide people with the skills to self-manage their condition and engage in exercise and functional activity. For example, patients go to coffee shops or libraries with pain management specialists to reflect and learn skills and strategies for everyday activities such as finding opportunities to stretch, remembering to pace or take breaks [41]. While CP physical rehabilitation increasingly focuses on function, current physical rehabilitation technology still focuses on exercise [40] or aiding relaxation [21]. While these aspects are useful, they often do not translate to increased confidence in doing functional activity [41]. For example, doing a neck exercise does not give people confidence in looking over their shoulder while driving.

\section{HOME REHABILITATION TECHNOLOGY}

There are very few examples of technology that supports people in doing functional activity in the home. Further, people have little motivation to do activity recommended by clinicians when confronted with the physical and psychological barriers imposed by $\mathrm{CP}$ and the demands of everyday life [18]. Thus, technologies for physical rehabilitation introduced to the home may have little or no effect on pain management strategies and situated practices [22]. Due to these limitations, designers are attempting to integrate technology within functional activity. However, technology designers do not fully appreciate user needs for rehabilitation, barriers to activity and the use of resources in the diverse home environment [3]. Most home-based studies on physical rehabilitation have focused on adherence to exercise. Some designers have attempted to highlight differences between clinic and home, and leverage the home environment and activity within it for exercise (e.g.,[4]), but did not aim to facilitate functional activity itself. Attempts have also been made to use technology to transform functional activities people enjoy or need to do into exercise to practise certain movements (e.g., $[5,6,8]$.

To motivate and support performance of everyday functional activities, designers have suggested the use of technology in quantifying functional tasks to enhance the feeling of doing (e.g., by using step counts [17]). However, they do not provide any real-time support in performing the activity itself. While post self-reflection on quantified activity is useful for self-management, it alone is not sufficient to overcome emotional barriers such as anxiety during performance of functional activities despite pain. Some technologies do provide real-time feedback but their focus is on correcting posture (e.g., [7] and commercial sensors such as Lumo Lift:[35]; iPosture :[28]). However, these approaches do not address psychological barriers (e.g., lack of confidence, pain, fear) to physical activity or support people in applying pain management strategies, vital for CP management. Thus, it is important to explore how technology can go beyond roles of control and quantification and support people with $\mathrm{CP}$ in discovering their own capabilities and applying and practising selfmanagement strategies for everyday functional activities.
REAL-TIME FEEDBACK IN PHYSICAL REHABILITATION Personalised multimodal real-time movement feedback is shown to be effective in supporting and motivating performance of physical rehabilitation exercises. It helps to guide and correct movement as well as enhance movement perception and achievements [29,33]. Most studies rely mainly on visual feedback as they are designed for in-situ exercise (often in front of a situated display). However, visual feedback may be unsuitable for supporting functional activity due to its ubiquitous nature. Vibrotactile feedback has been effectively used in sports and other movement related activity $[9,43]$ but it may be difficult to perceive when engaged in other activity or uncomfortable over a long duration. Thus, it may be better for signalling specific events rather than providing continuous feedback [15].

Auditory feedback has been effective in motor rehabilitation (e.g., $[38,45])$ and explored in both situated and mobile contexts for initiating and guiding movements, facilitating coordination and improving motor performance. Sonification (mapping movement into auditory feedback), has been used to facilitate movement training in many scenarios: e.g., after stroke or spinal cord injury [38,47], to guide target movements using electromyography data [26], to transform 3D movement analysis of shoulder joint kinematics to correcting therapeutic exercises or posture [45] and to support coordination in patients with poor proprioception [20,27]. Poor proprioception is a relevant problem that also affects people with CP [34].

Recently, movement sonification has been used to support people with $\mathrm{CP}$ in addressing psychological barriers when performing feared movements [42] using a device designed iteratively with patients and physiotherapists; the device was further informed by observations of pain management practice and analysis of individuals' needs in real life environments. This study showed how self-calibrated movement sonification feedback could lead to increased movement awareness, performance and confidence in people with $\mathrm{CP}$. Thus, real-time feedback of people's movements during functional activity could potentially make them more aware and confident of their capabilities. However, this has not been investigated yet. In this paper, we identify the opportunities offered by real-time movement tracking and feedback technology to address the functional needs of people with CP. Before presenting our study, we present the design of the device used in our study.

\section{THE DEVICE}

We used a modified version of the device proposed in [42] to track and sonify movement in CP. This device was found to be suitable for a ubiquitous context and people with $\mathrm{CP}$ had suggested its suitability for supporting functional activity. In addition, the device was designed with the support of people with $\mathrm{CP}$ and physiotherapists and validated in exercise contexts. However, the focus of this study is not to further validate this device, but to use it as a research tool [49] to understand how sensing and feedback 
technology can support functional activity in CP. We iteratively refined and tested the device with five people with CP (two males and three females from 28 to 64 years of age, with CP for 6 to 31 years) to improve its usability for longer self-directed home use. While the device was redesigned and additions were made (e.g., remote control, and interface to record pain levels), the design concept, sonifications and calibration approach were the same as in [42] and are briefly described below.

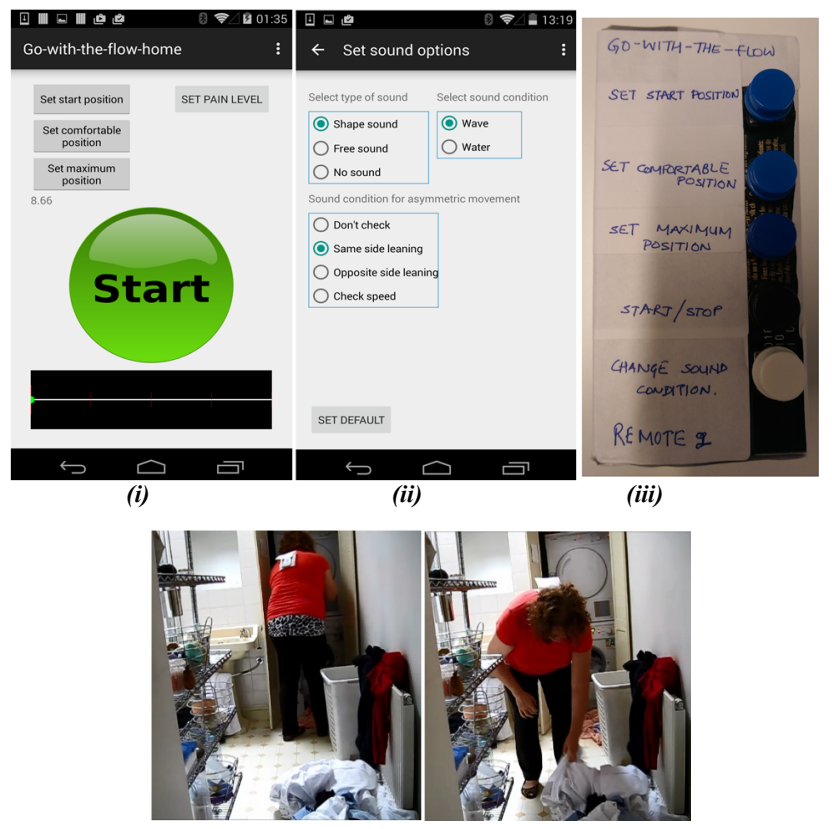

Figure 1: (Top) Smartphone App interface - two tabs: (i) three calibration buttons, self-set pain option and start sonification button and (ii) sonification options (iii) Bluetooth remote control interface. (Bottom) Still pictures from participant's video-diary wearing the device while doing the laundry.

The device (Figure 1) included: an Android Nexus phone, a sonification app that we designed based on [42] and a remote control. Participants were also provided with two tshirts, with a pocket stitched on the back for the smartphone (see Figure 1 - Bottom). A smartphone armband was provided to wear the device on other body parts. The device (using smartphone movement sensor through an app) worn using either the t-shirt or the armband tracked the relevant body part. Our focus in this paper was mainly on trunk movements as we were interested in chronic back pain.

The smartphone gyroscope tracked the body part movement (amount of rotational movement along one axis) and transformed it into sound. For the sonification to be effective, users needed to calibrate the mapping of movement to sound in a way that addressed their physical and psychological needs. To do this, they would first explore their movement while wearing the device and set three points associated with particular body positions within the desired body movement. For example, to calibrate the device to support trunk stretching forward movement, the smartphone would be worn on the trunk and the person would set three trunk positions: (i) a standing vertical position (start position), (ii) stretching forward to a point where they felt confident despite the pain (comfortable position), and (iii) a maximum stretching position they wanted to achieve (maximum position). To set the position, the person would stretch the trunk accordingly and press the appropriate button on the remote control. The smartphone would then record the position, i.e., the degrees of bending of the trunk. Voice feedback was used to confirm the registration of each position (e.g., "start position set").

The device used the set positions as points where the sound changed. Thus, when the person moved while wearing the calibrated device, the changes in sound at those points provided information about body position while moving and of progress. As in [42], the wave sound consisted of seven equidistant ascending tones before the comfortable point followed by four equidistant descending tones up to the maximum point (see Figure 3a). After that point the sound would flatten out, i.e., the same tone repeated as the person stretched or bent beyond that point. The sonification was played in reverse as the person moved backwards. In the water sound condition, reaching the comfortable point was signalled by a splash sound while any other movement generated the sound of moving water. The windchimes sound did not use the set calibration but simply transformed movement into windchimes sounds. We included the wave, water, windchimes sounds in the app as these sounds were the most useful in [42]. We also included the possibility for no sonification (i.e., just tracking).

Through the app interface or the remote control (Figure 1 top) the person could recalibrate the sonification to their physical and psychological capabilities at any time (e.g., based on perceived physical capabilities or increased pain). The remote control facilitated access to the calibration and sound options when the smartphone was worn on the trunk (Figure 1, third photo). It was deliberately designed as a rough low-fidelity prototype cardboard box with click buttons and handwritten labels so users would not hesitate to suggest refinements [16]. Users could also self-report pain levels on a scale of $0-10$.

\section{HOME STUDY}

We ran an in-the-wild study to investigate the use of the device during self-managed everyday activity [31]. We wanted to understand how a device providing run-time feedback about people's own movements would facilitate a variety of everyday activities as contextual usage patterns cannot be discovered in a one-off encounter during a control study $[25,32]$. Besides, the novelty of a new technology wears off after a certain period of time, and after using it for longer people can critique different aspects of it than they do on a first encounter [32].

\section{Participants}

People were recruited through the project website (www.emo-pain.ac.uk) and social media. Potential participants were informed about the study on contact and 
gave consent for using the device at home. People were instructed on the use of the device before it was given to them for home use. All selected participants were smartphone users. Our studies were approved by the University Ethics Committee.

Table 1: Participant profiles for long-term study.

\begin{tabular}{|c|c|c|c|c|c|c|}
\hline ID & Age & Pain area & $\begin{array}{c}\text { CP } \\
\text { duration }\end{array}$ & Still at work? & Gender & $\begin{array}{c}\text { Lives } \\
\text { with }\end{array}$ \\
\hline P1 & 59 & Back, foot & 21 years & $\begin{array}{c}\text { Works from } \\
\text { home }\end{array}$ & Female & Partner \\
\hline P2 & 54 & Neck, back, hip & 18 years & $\begin{array}{c}\text { Community } \\
\text { Volunteer }\end{array}$ & Female & Partner \\
\hline P3 & 43 & Back, groin, leg & 2.5 years & Not employed & Female & Alone \\
\hline P4 & 34 & $\begin{array}{c}\text { Neck, shoulder, } \\
\text { lower back }\end{array}$ & 6 years & Nursery worker & Female & Partner \\
\hline
\end{tabular}

Four people with CP participated in the study (see Table 1) and used the device in their everyday life (mainly home). We set an initial one-week period. The device was given to one participant at a time so that we could improve the design between participants and provide support if needed. However, some participants asked to keep the device for longer which was agreed. Whilst only four, these participants represent four diverse case studies in terms of length of CP condition, the amount of mobility they felt capable of and the type and level of activities within and outside the home:

$\boldsymbol{P 1}$ is a writer and works from home. Her daily routine consists of writing at her laptop, housework, stretching and walking. She does weekly Pilates and occasionally volunteers in a community kitchen. During the second week of the study, she used the device on holiday with a friend. She wanted to use the device to increase her awareness of everyday movements in the house and while writing.

$\boldsymbol{P 2}$ is retired and does occasional volunteer work. Her daily routine consists of housework and stretching exercises and weekly gardening. Her motivations for using the device were to explore her activities at home and practise stretches.

$\boldsymbol{P 3}$ does not work at present. Her relatives and friends visit often and she has an active home social life. Her daily routine consists of housework, occasional shopping and visits from friends. Her initial motivations to use the device were to increase awareness of her movements and posture, and to avoid positions/movements that caused her pain.

$\boldsymbol{P} 4$ works as a nanny. Her daily routine consists of looking after a child (including taking her out), cleaning, tidying, and cooking. She tries to incorporate stretching between tasks. Her initial motivations to use the device were to learn more about her movements to help reduce her pain.

\section{Procedure}

On the first day, participants were introduced to the device. They were taught to calibrate the device for a start position, comfortable stretch and maximum stretch of the trunk during the forward reach exercise. After exploratory stretches using different sound options, we asked how they expected to use the device to support daily activities and exercise and what their motivations for using the device were. Participants could use the device as they liked during activities around the home or outside and during exercise. There were four interviews per participant: (i) on the first day of the study before using the device to explore expectations from use, (ii) in the middle of the study (third or fourth day) for experiences of use, (iii) at the end of the study when the device was collected for reflections and changes due to device use, and (iv) a follow-up interview after a week to ask people about any other insights about using the device. Interviews lasted between 15 and 30 minutes. Participants were also given a paper diary to record their thoughts and experiences of using the device during functional activity or exercise. We asked them to record where they thought the device had potential to facilitate activity or was not useful. One participant also used a video diary (Figure 1 - Bottom). Participants were also invited to record their pain level daily or as needed. Interviews were audio-recorded and the researcher took notes regarding the activity, the physical environment of the home and where the activity was situated within the homespace. Participants were encouraged to email/phone for support and the researcher was in frequent contact with the participants during the study to address any concerns. We finally ran a post-study interview with a CP specialist physiotherapist ( $>10$ years pain management experience) cued by the analysed data (interviews, diary entries, sensor data and videos) collected in the home study.

\section{Data Analysis}

The calibration and movement data from phone sensors was logged in a file with a timestamp. We used Excel software to analyse data to explore how people calibrated and used the device over the study period for physical activity. People's self-reported pain levels were captured to invite reflection on how pain affected the use of the device and activity levels. Diary entries were analysed in conjunction with tracked data to make sense of calibrated points with activities so we could see if people used any calibration strategies to facilitate the activity. Interviews gave further insights into strategies and reflections on device use. We did not aim to do an in-depth analysis of the sensors data as it was not possible to draw conclusions about people's activities based only on analysis of sensor logs. However, based on the timings of the calibration and the notes in the diary, we extracted a few activity $\log$ s for illustration of how people used the device to create strategies for activity and how they used and changed calibration for particular activities. All interviews were transcribed and we did a thematic analysis to identify patterns and themes [12].

\section{RESULTS}

All participants used the device for no less than a week with varying number of minutes per day as shown in Figure $2-$ Left graph: number of days indicated in brackets on the $\mathrm{x}$ axis). P2 asked to keep the device for 2 weeks but in the second week she used the device only for two days due to 
illness. All participants reported mainly using it to sonify trunk movements during functional activity but also tried other parts of the body (e.g., to train leg movements in sitto-stand). People varied in their range of reported painlevels (Figure 2 - Right) and pain levels varied daily (See P1's daily pain self-report in Figure $3 \mathrm{~b}$ - bottom graph).

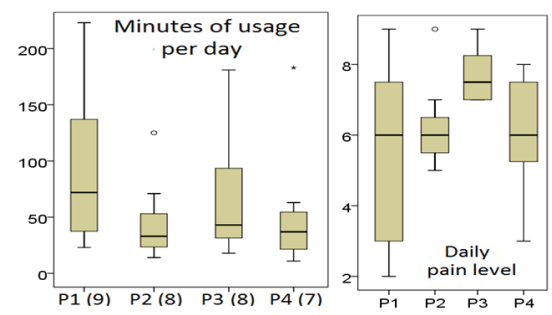

Figure 2: (Left) Daily usage of the device by each participant. Number in brackets indicates the number of days the device was used. P2 kept the device over 14 days but used it only over 8 days due to illness. (Right) Daily self-reported pain.

The analysis of the device usage (data from device) shows the emergence of a set of calibration strategies to facilitate functional activity. For example, Figure 3b (top graph) shows P1's daily recalibration of the values for the comfortable (green) and maximum (blue) position of the bending of the trunk. The values were used to recalibrate the sonification on the device (e.g., the wave sonification as illustrated in Figure 3a). The minimum point is not reported as it is always around 0 degrees (standing position). We used the diary data filled by each participant to associate each recalibration setting with the functional activities it aimed to support (see Figure 3 b-c).

The main calibration strategies that emerged are discussed next within the six themes that emerged: (i) body awareness and self-management skills, (ii) support beyond functional activities, (iii) disrupting routine and challenging habits over time, (iv) providing a sense of control over the environment, (v) opportunities for setting boundaries and design, and (vi) overcoming social constraints. We also highlight the affective dimensions within each theme.

\section{Body awareness and self-management capabilities}

People used calibration strategies to increase their body movement awareness during functional activity. The information emerging from the calibrated sonification was used to help in managing their physical and psychological resources and recognise their capabilities. Once participants had established that the sound feedback truly reflected their movements through exploration, they trusted the information provided by the device and associated the feedback with their own movement. The feedback provided them with an enhanced awareness of their body, making their body "reappear from behind the pain". This reappearance enabled them to focus on their movements and capability rather than on their pain.

All participants started by calibrating their movement for a trunk stretching exercise based on their perceived capability. The term "capability" is used here to indicate what people perceived they could do without exacerbating pain or anxiety. All participants, (except P4) found that their perceived capability was less than what they could achieve during the same movement in functional activity without increasing anxiety or pain (see circled points in Figures 3c and Figure 4). For example, P1 stated "The first thing I realised after calibrating the device, was when I went to get something from the shelf, I went past my calibrated maximum without thinking but the sound told me that I was beyond my maximum. So, I can do more than I think I can. It's the fear that the pain will kick in that holds me back. But the sound immediately gave me that insight into the way I move when I am thinking about it and when I'm not." P3 said, "Just pottering around and hearing the sounds, you realise you are moving and stretching a lot more than you think you are!' The interviewed physiotherapist felt that increased awareness of capability was an important use of the device, congruent with the literature on $\mathrm{CP}$ that shows that people may be afraid of making certain movements but can do them in a different context when not focused on them [37].

Two types of calibrations emerged in our study: (i) calibration for exercise (CE) based on perceived capability, and (ii) calibration for functioning (CF) based on not just perceived capability but also the movement required by the activity. When the functional activity required bending beyond perceived bending capabilities and hence beyond $\mathrm{CE}$, the flat sound information triggered anxiety as it indicated going beyond their maximum. However, after initial use of the device, most participants calibrated the sonification to the functional activity rather than perceived capability and used the changes in sound as information (discussed later in detail). The calibration of the comfortable point was generally placed half way between the starting and the maximum position, based on what people felt they could achieve. Figure $3 b$ (top graph) and Figure 4 show the recalibration values for each participant ordered by the maximum calibrated position they set and the associated functional activity. The sonification also shifted people's focus to pleasurable sensations such as the sound of tones or windchimes. For example, P3 said " $I$ wouldn't just bend for exercise, I find bending uncomfortable and painful. But I need to bend - my dishwasher, washing machine, cleaning my bathtub all need me to bend - and these sounds help me to focus on [sounds] instead of the pain. That is helpful".

The information provided by the sound and the increased body awareness made people feel more willing and confident of applying self-management skills. For example, the calibrated sonification supported people in generalising certain movements to different contexts, which may have been anxiety-provoking previously. P1 stated: "It's probably good for my back to lean further forward but I'm hesitant about doing it. I find reaching for the washing machine painful. However, I realised when doing exercise with the device and hearing the sounds, I could use the roll- 
down [Pilates exercise] to load the machine, which relieves my back. I do the roll down all the way down [to load the washing machine] when doing exercise and I'm nowhere near the bottom when this tells me I've reached my maximum [i.e., the washing machine target]. So, it's useful for trusting my body more than I do."

Further, as the wave sound was designed to increase awareness of even small movements, people used it to learn movements and increase awareness of movement strategies (e.g., pacing, speed). Indeed, when the functional activity required less bending than they felt capable of, they calibrated the sonification to the smaller movement in order to maximise the information obtained and better control their body. The information provided by small sound intervals in the wave sound, made people feel rewarded and perceive the activity as worthwhile. P3 said, "It's nice to hear the sounds, even when I am only doing small things around the house I feel like I am achieving something".

People indicated that in some activities there may be a subspace that is more important to them and needs more information (e.g. P1 recalibrated the device during writing, as the initial bend angles were more important to her.) Figure 4 shows how people adapted the feedback to their activities: e.g., P2's data when stretching for gardeningweeding vs gardening-bending to pick up garden waste. The duration of the activity also mattered. For long duration activities, people reported using smaller intervals to remind them not to overdo (e.g., smaller interval in P1's data when writing and in P2's data for weeding). The physiotherapist felt that the calibration and sound feedback could be very useful in pain management as "people can play with their movement and see patterns of what affects them - that they can do more or they need to take breaks, or whatever. It's the difference between seeing it and being told ..."

\section{Support beyond functional activities}

Device feedback was interpreted based on context. In some contexts, the device was companionable. For example, P3 said, "I used it while gardening and it was very pleasant to have the sounds as company as I pottered around. It made me aware but also was a pleasant companion." In addition to using the wave sound as information, she enjoyed the wind chimes sound which she felt encouraged movement without focusing on targets. She suggested using a favourite song or soundtrack that would play only if the person was moving, thus encouraging movement without target information. People used functional activity to do stretching exercises and calibrated the device to exercise needs. For example, P4 calibrated the device to stretches and pushed the swing with the toddler she looked after to do reach forward exercises. While there was no target to achieve in this activity, she felt supported by the sounds which would inform her if she was going past her pre-set maximum. The physiotherapist agreed with this approach where the person did not focus on pain levels to guide activity since pain levels fluctuate leading to large variations in activity; rather activity levels were based on perceived capability. He felt that the sound supported the person in engaging in "playful movement".

Using sound in a (co) supervisory role to overcome barriers In other contexts, especially when there was high cognitive load, participants felt that the sound was reassuring and informative and used it in a supervisory capacity. For example, people suggested that the device could be used to practise suggestions given by physiotherapists such as "not leaning too far back" (P3) when stretching. Others reported that they were reminded to: "keep changing position" and "do more stretches when standing for long periods" (P1), "remember not to tuck legs under" (P3), "sit straighter" (P2) and "move while doing the dishes" (P2, P4). In this case, the device could provide "a second set of eyes" (P3) and help people to have "more control over their movements" (P2). P2 explained, "what is helpful is there's like a bit of that kind of 'physio on your shoulder', type of thing, to tell you or to remind you about doing that movement." For example, P4 realised her lack of movement while washing the dishes because she got no sound feedback even though the device was on her back and set to an audible sound condition. This is often the case in people with $\mathrm{CP}$ as their muscles tighten up [23] to support the body leading to increased pain. P2 also reported this lack of movement in her diary "Cleaning teeth, leaning over basin at, example a 30 degree angle, for a sustained period (even 2 minutes) is worse than leaning forward to retrieve something." The physiotherapist said, "this is like pacing activity: we highlight this in pain management programmes so they do not overdo activity. The reminder is helpful but also that they have to be aware of it to set it themselves."

The supervisory role of the device also increased people's awareness of their adapted or protective movement habits. For example, P2 habitually leaned over her walking stick when walking. She always asked family and friends to highlight this but she was not aware of it when on her own: "if you're walking with a stick and you lean forwards too much, then that can trigger pain. So, things that remind you: I've often thought that it could be really handy."

\section{Disrupting routine and challenging habits over time}

People used the device feedback to change their routines. P1 and P4 introduced stretches when washing the dishes. P4 reported that she felt less stiff after doing dishes if she incorporated stretches during the activity: "I don't realise that my muscles are starting to be stiff until I am in pain." At the same time, the participants felt that the device could help them to form new habits. In some cases, even if participants switched off the sound feedback from the device because they wanted to focus on something else, they found that the presence of the device between their shoulders made them aware of their posture or movement. P1 said, "What I also found really interesting is that when the thing had switched off, just having it between my shoulder blades made me more conscious of my posture." A 
week after they had stopped using the device, people reported continuing with new habits related with posture and movement, not just due to the initial awareness built by the sound feedback but because of new awareness that continued to emerge and helped to make changes (such as interspersing stretches in activity). They reported feeling more "body-aware" (P4) after the study and that they could discuss the device feedback on existing habits with their healthcare providers/partners or choose to change them. People felt the device also highlighted when they were avoiding a movement. The feedback from the device supported them in moving more than they would otherwise have done, especially in anxiety-provoking contexts. "It's telling you that you have more mobility than you think you have..." (P1). However, to form habits behaviours need to be repeated over a long time. The movement sonification possibly facilitated this process by enhancing awareness. Even though our study was short to evaluate if habits had been formed, participants reported more confidence in movement and greater awareness of their body. P3 said, "My friend said, 'something about you has changed. You're moving so much better.' And I feel that, I do. I don't think as much because I know how much I can do".

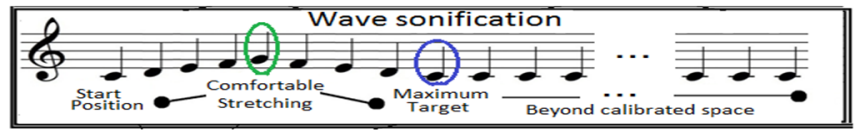

(a)

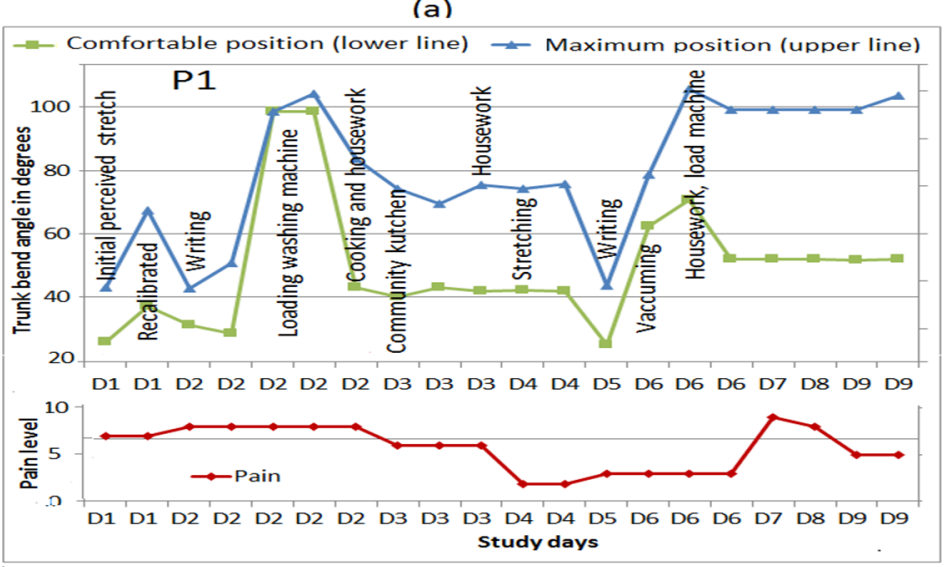

(b)

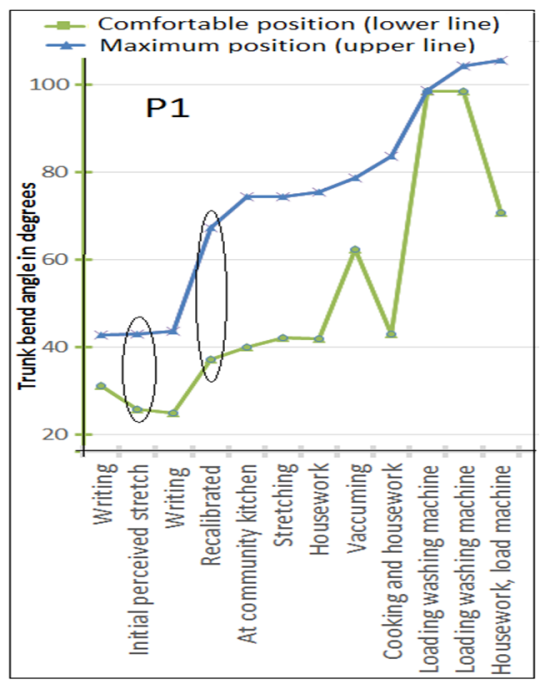

(c)

Figure 3: (a) Wave sonification with comfortable and maximum positions highlighted. (b-bottom graph) P1's daily self-reported pain levels. (b-top graph) P1's daily calibrated comfortable and maximum trunk bending positions. (c) P1's daily calibration points organised from smaller to larger maximum position (only points clearly associated with activities are reported).

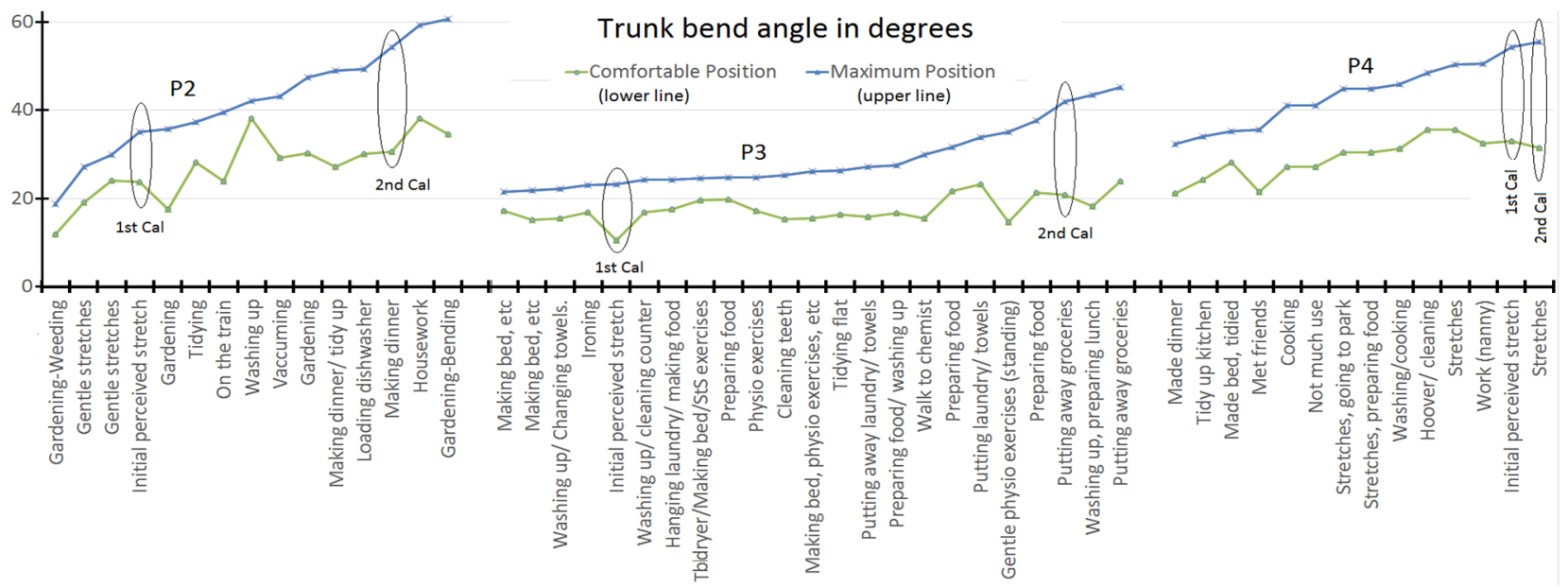

Figure 4: P2, P3, P4' daily calibration points for trunk bending degrees organised from smaller to larger maximum position boundaries (only points clearly associated with activities are reported). $1^{\text {st }}$ and $2^{\text {nd }}$ calibrations values circled.

When the device was used over an extended period during our study, the nature of use changed over time. For example, in the beginning the most informative sound feedback (wave) increased awareness of body movement during exercise and function. Functional use of the device allowed people to generalise that learning across activities and contexts to address barriers to movement in $\mathrm{CP}$ such alleviating anxiety (e.g., P1 using the washing machine). 
Participants reported about the wave sound notes: "after a while the sounds get quite annoying" (P3). This could affect long-term use of the device as "people like me [with CP] can be more sensitive to certain sounds." This finding resonates with the concept of sensitisation in the psychology literature, where repeated exposure to a sound can make it seem unpleasant [30]. P3 suggested the use of a lower pitch with a bass instrument. Despite this, participants preferred the wave sound to other sounds for building awareness due to clear information and transitions between notes. Participants suggested that after learning/developing an understanding of a movement, they could switch to cued feedback (feedback at certain predefined points during movement). A second suggestion was to use vibrotactile or visual feedback instead if a person had started to feel tired of the sounds or was in a situation where sound could be disruptive or not socially acceptable.

\section{Providing a sense of control over the environment}

While most of the device use was focused on raising awareness of their own body, some participants also identified aspects of their environment that could be changed for better functioning. For example, P3 used the device to reorganise her space to function more efficiently. "Realised my kitchen towels are hung too low, which necessitates regular bending. Now hung higher."

While this could be viewed as an avoidance strategy[24], P3 reported that reorganising her space helped to conserve energy for other enjoyable things that she otherwise could not do. The interviewed physiotherapist reiterated that, "pain management is not about pushing past pain but about moving often and building slowly." So, reorganising to avoid overdoing could help people to manage their activity. Other participants used the setup of their environment to make tasks challenging, such as keeping things out of reach so they would have to stretch to use them. However, one difference between the two cases is the regularity with which the task needs to be performed. If a task is performed repeatedly, then people may be spending a lot of energy in carrying out the task and managing their resources.

\section{Opportunities for setting boundaries and design}

Most participants found that the sonification was reassuring and engendered a feeling of trust in their body capabilities. At the same time, they felt that the calibration could be in part transferred to the device. For example, P1 wanted the device to suggest additional points to raise awareness of her actual capability and to suggest activity increments, hence overcoming her fear and hesitation in making those increases. She said, "You set your initial point, comfortable position, maximum position, and have [sound] feedback. When you're doing movements, if you consistently reach another point, have it save that and feed that back to you. [...]. Now if the device spotted that we consistently get to this point beyond our maximum, have it store that point and give you the option to see how far you can go, [...] a kind of 'well done' sound that could help in recalibrating next time and you can opt to set it to that further maximum. And then you can get beyond that!"

However, there could be problems with this approach: P3 found that stretching further in functional tasks did not always indicate higher or increased capability. P3 said, "I have no choice sometimes. If I drop something, I have to bend to pick it up as I live alone but that does not mean it is not a painful movement and during the day I may have a few of those.' $\mathrm{P} 2$, for example, started associating certain sounds with pain, because when she was doing some tasks that pushed her past her limit, she felt pain and started associating the note that sounded at that point with pain. She said, "Although the app helps as a reminder to control movements more, it sometimes also makes me tense up more than I normally would."

Some participants felt that using the device with a physiotherapist could help them to develop calibration and sonification strategies. However, the interviewed physiotherapist felt that the context in which an activity is performed influences the calibration and hence it may not always be useful to calibrate a movement in hospital settings to be used in the home during functional activity.

\section{Overcoming social constraints}

All participants used the device outside the home in different situations, such as on the bus or train (P2, P3), meeting friends at a shopping centre (P4) and volunteering in a community kitchen (P1). We discovered that social use of the device was different (i) in presence of strangers, (ii) with acquaintances and (iii) with friends and family.

In the presence of strangers, in situations such as on the bus or train, participants felt that they would find the device useful to remind them to change position or stretch, which they often neglected to do not just because of pain but also because of social anxiety. Participants were not concerned about strangers noticing the device itself in public. "Everyone's busy, no one really notices" (P4). However, they felt that the sound feedback could attract attention to them and, more importantly, to their condition and cause embarrassment. Some participants used the device with the volume turned down in public. All participants felt that vibrotactile feedback would be useful in public places. Headphones were not a viable option as P1, P2 and P4 preferred to be more mindful of their surroundings.

With close friends and family, people liked that the device and the sound invited discussion. For example, P1 used the device to discuss how pain affected her movements with her partner and a friend. P4 wore it during a trip to the shopping centre with her friends to show and discuss how she planned to use the device. However, in more interactive contexts, sound feedback distracted people and interfered with conversations. They stopped using the device in these situations or turned off the sounds. With acquaintances, people felt that the sound invited too much attention forcing them to share details of their CP condition (e.g., P1 in the 
community kitchen). Vibrotactile feedback for cueing significant events would be more suited to these situations.

\section{DISCUSSION}

Our study investigated how wearable movement sensing and feedback technology can help people with CP to engage in everyday activities. In chronic conditions such as $\mathrm{CP}$, most everyday activity as people's physical and psychological resources can be limited [18]. Our study demonstrates that technology has the potential to facilitate everyday functioning, beyond an assistive capacity (i.e., compensating for limited physical capabilities (e.g.,[34])). We argue that technology can be an enabler of functional activity by facilitating exploration of body capability, enhancing body awareness, and providing the tools and the skills for applying personalised movement-related pain management strategies. However, the way feedback is used to facilitate functional activity differs from its use in supporting exercise. Here we discuss three main findings that emerged from our study: the role of wearable technology in enabling functional activity, feedback calibration strategies in relation to function, and active role for technology during automatic calibration.

\section{Wearable technology roles in functional activity}

There is evidence that movement sensing and real-time feedback can increase body awareness, provide movement guidance (e.g., pacing) and work as reward during exercise $[33,42]$. Our study shows this is also the case for functional activity. When in a wearable form, such device can support people everywhere and not just in front of a display. It can, thus, help people to better understand their body movement directly in relation with functional tasks. In our study, people reported increased movement awareness, increased confidence to develop or apply movement-related pain management strategies (e.g., pacing, rolling down) and in doing activity as their capability to do so is reinforced by sound feedback. We argue that this increased confidence is due to two factors: (i) self-calibration of sonification: knowing that the movement is within self-set boundaries and that changes in sonification reflect specific positions and progress), and (ii) an embodied perception of the feedback (perceiving feedback as one's own body).

Current technology relies on people's reflections on their capability when transferring physical gains in exercise to everyday life ignoring the context of movement [29]. Such design is generally based on behavioural change theories that emphasise goal setting and rewards for motivation [29] without considering interference from emotional barriers. Given the emotional distress people with $\mathrm{CP}$ have to deal with while engaging in demanding activities and possibly a poorly functioning proprioceptive system, simply reflecting on similarities between movements can be challenging [34]. While technology for exercise is effective and needed, it is not sufficient for rehabilitation for daily living and thus needs to be complemented with technology that enables a finer level of perception of one's body and context.
Indeed, calibrated run-time movement feedback acts as a bridge that connects the physical experience of doing activity and perceived capabilities. Moving within self-set sonified boundaries gave our participants confidence to build on past successes and extend the capability to other contexts ("I loaded the washing machine yesterday within these boundaries, so I can do that today and I can do a similar movement in another context"). The trust built by the sonified experience of bending to a certain point can facilitate progress or alternatively incremental exploration of different contexts. Indeed, our findings show that people tended to under-estimate their capabilities in the first exercise-based calibration, but by trusting and owning the sound as a reflection of their body movement, they started exploring larger ranges of calibration and movement. In addition, some participants reported that once they built trust in their body based on the external sound representation that reinforced their proprioception, they felt more confident even without sound feedback. This is different from quantified-self technologies which provide just information (e.g., step counting [17]). The direct experience and perception of the body bending (rather than a number) helps to build confidence and a sense of being able to listen to one's body. Further, we argue that beyond self-calibration, the fact that the device was wearable rather than a distributed set of video-cameras and feedback actuators around the home, help to build trust the meaning of the sonification feedback as the person moved and performed similar movements in different contexts and tasks. While a wearable may not be the only way to reach a connection between feedback and body, it is an important factor to consider when designing for psychological barriers in functional activity.

\section{Feedback calibration strategies}

Our study shows that the role of technology can go well beyond simply correcting posture and movement trajectory (e.g., $[7,28,35])$ and instead support active development and application of self-management strategies. Indeed, another important finding that emerged from our study is how to calibrate for functional activity. In comparison with exercise or assistive technology $[38,42]$, our participants generally calibrated the feedback to reflect the functional activity range of movement rather than their own capabilities. In some situations, a smaller range of calibration was preferred even within specific functional activities. For example, for long duration activities, overdoing was a concern as extended exposure to the same movement could lead to fatigue and increased pain.

In previous studies with people with CP $[42,17]$ suggested using functional activity to measure exercise progress. Instead, what emerged in our study was that functional activity was a space for exercising. However differently from [8], it was not just about simply using pleasurable activities but exercising capabilities needed in challenging everyday activities. Beyond using sonification and calibration to develop strategies to address challenges, 
smaller calibration ranges were used to increase information within a specific part of the movement that the person wanted to explore. This finding highlighted the importance of being able to recalibrate movement ranges not just based on a person's capabilities but on the demand and purpose of the activity, and on the level of body and movement awareness to develop.

While the sonification of the maximum target was used to create a sense of achievement and to avoid overdoing, sonifying the complete movement as the person was performing it was critical. By perceiving the full movement, people could learn about their body and how to listen to it, apply pain-management strategies and even develop routines. This suggests that current everyday sensing and feedback technology for CP designed to detect wrong posture $[7,28,35]$ or quantified activity (e.g., [17] should be augmented, where appropriate, with full movement feedback to create a space for learning self-management and consequently behaviour changes. Indeed, our participants, continued to develop routines and strategies even when they stopped wearing the device. The calibration points could be designed to facilitate body awareness and development of strategies beyond helping with executing the activity at hand.

\section{Active role for technology during automatic calibration} Clear needs and opportunities for automatic calibration of the technology emerged from our study. Technology can offer calibration options as it gains information such as the person's capabilities, context, and routine. Some people felt that this would be useful. The device could be modified to store movement ranges for certain activities and could suggest increments to the user when sensing increased capabilities. However, previous studies [31,42] and our findings suggest that the exploration of one's body and building trust were critical steps to learning and even developing pain-management strategies.

Rather than the device taking full control, more engaging ways could be developed to treat calibration as a dialog between the device and the person for better learning about one's body. Such a dialog can address emotional barriers where people may be avoiding increasing their range of activity for fear of increased pain or damage. If the device detects that the person has not recalibrated for a while even though their capability is improving (for example, by tracking fluidity of movement, lack of anxiety when doing movement [2]), it could suggest a change in calibration. Conversely, if people move a lot and get regular flare-ups or tiredness due to overdoing $[2,36]$ (indicated by larger rest periods), the device could suggest a reduced calibration.

Beyond supporting calibration by using movement history, the device could support better calibration and suggestion for activity engagement, by gathering information about the house. With the emergence of smart homes [48] and activity recognition technology [14], we can foresee a dialog between the wearable device and the sensors in the home. In this context, the device could gather information about the space in which an activity is performed, the type of activity performed (e.g., sensing that the person is in the kitchen and $\mathrm{s} / \mathrm{he}$ bending to load the dishwasher) and inferring information about duration to let the user know about overdoing. Such rich information would help the device to provide insightful advice for effective support.

Finally, we acknowledge that the results of our study cannot be directly generalised to other people with $\mathrm{CP}$ due to the small sample size. However, we provide a different perspective to physical rehabilitation technology, i.e. to support functioning. In addition, our study demonstrates the importance of an idiographic approach for real-time feedback to enable self-management during functioning. As such, this study has laid the foundations for a larger study to better appreciate variations in strategies and to support people with CP to develop and apply their own strategies to self-manage activity. Further, previous work in other conditions (e.g., MS, stroke) has largely focused on exercises or walking rather than complex functioning $[1,10]$. Our results suggest that our approach could extend to other populations that also experience fluctuating pain, difficulties in physical function, low confidence in movement capability, anxiety about physical activity, difficulty in transferring improvement into better general function (e.g., [11]). However, a further study is needed to understand the generalisations feasible given differences between conditions.

\section{CONCLUSIONS}

Our study investigated the opportunities offered by wearable sensing and feedback technology in facilitating functional activity in the home. Building on previous studies, we adapted a smartphone-based device for a 1-2 week-long home study with people with CP. Four case studies were used to present different functional activities, capabilities, and strategies for device use. Our findings showed that the real-time sonification of functional activity movements can increase awareness of capabilities, selfefficacy and confidence. Through in-place active awareness and reflection, rather than post (out of context) reflection, self-calibrated sonification can support application of movement-related pain management strategies. Our study also showed how people developed sonification strategies to address their needs within the challenges posed by the functional activities and that these strategies differ from the ones for exercising. The findings also provide initial insights into the role of different types of feedback, particularly aural vs. tactile. Finally, while opportunities for automation of calibration emerged, it is clear that selfcalibration is beneficial to learn about one's body and build confidence in functional activity.

\section{ACKNOWLEDGEMENTS}

EPSRC EP/H017178/1 grant: Pain rehabilitation: E/Motion-based automated coaching. www.emo-pain.ac.uk. Thanks also to study participants for their time. 


\section{REFERENCES}

1. Deepti Aggarwal, Bernd Ploderer, Frank Vetere, Mark Bradford, and Thuong Hoang. 2016. Doctor, Can You See My Squats?: Understanding Body Communication in Video Consultations for Physiotherapy. Proceedings of the 2016 ACM Conference on Designing Interactive Systems (DIS'16). 1197-1208.

2. Ming Hane Aung, Stephan Kaltwang, Bernardino Romera-Paredes, et al. 2016. The automatic detection of chronic pain-related expression: requirements, challenges and a multimodal dataset in IEEE Trans. Affective Computing 7, 4: 435-451.

3. Lesley Axelrod, Geraldine Fitzpatrick, and Jane Burridge. 2009. The reality of homes fit for heroes: design challenges for rehabilitation technology at home. Journal of Assistive Technologies 3,2: 35-43.

4. Naveen Bagalkot and Tomas Sokoler. 2011. ReHandle: towards integrating physical rehabilitation in everyday life. CHI'11 Extended Abstracts on Human Factors in Computing Systems.

5. Naveen Bagalkot and Tomas Sokoler. 2012. Unboxing the tools for physical rehabilitation: embracing the difference between the clinic and home. Proceedings of the 7th Nordic Conference on Human-Computer Interaction: Making Sense Through Design. 597-606.

6. Naveen Bagalkot, Tomas Sokoler, and Riyaj Shaikh. 2012. Integrating physiotherapy with everyday life. Proceedings of the Sixth International Conference on Tangible, Embedded and Embodied Interaction 91-98.

7. Naveen Bagalkot, Tomas, Sokoler and Suraj Baadkar. ReRide: Performing Lower Back Rehabilitation While Riding Your Motorbike in Traffic. 2016. Proceedings of 10th EAI International Conference on Pervasive Computing Technologies for Healthcare

8. Madeline Balaam, Stefan Rennick Egglestone, Geraldine Fitzpatrick, et al. 2011. Motivating mobility: designing for lived motivation in stroke rehabilitation. In Proceedings of the SIGCHI Conference on Human Factors in Computing Systems (pp. 3073-3082). ACM.

9. Karlin Bark, Emily Hyman, Frank Tan, Elizabeth Cha, Steven A. Jax, Laurel J. Buxbaum, and Katherine J. Kuchenbecker. 2015. Effects of Vibrotactile Feedback on Human Learning of Arm Motions. IEEE Transactions on Neural Systems and Rehabilitation Engineering 23, 1: 51-63.

10. Athina Belsi, Enrica Papi, and Alison H McGregor. 2016. Impact of wearable technology on psychosocial factors of osteoarthritis management: a qualitative study. BMJ Open 6, 2: e010064.

11. Valerie A J Block, Erica Pitsch, Peggy Tahir, Bruce A C Cree, Diane D Allen, and Jeffrey M Gelfand. 2016. Remote Physical Activity Monitoring in Neurological
Disease: A Systematic Review. PloS one 11, 4: e0154335.

12. Virginia Braun and Victoria Clarke. 2006. Using thematic analysis in psychology. Qualitative Research in Psychology 3, 2: 77-101.

13. Harald Breivik, Beverly Collett, Vittorio Ventafridda, Rob Cohen, and Derek Gallacher. 2006. Survey of chronic pain in Europe: Prevalence, impact on daily life, and treatment. European Journal of Pain 10, 4: 287-333.

14. Tanzeem Choudhury, Borriello Gaetano, Sunny Consolvo, Dirk Haehnel, Beverly Harrison, Bruce Hemingway, Jeffrey Hightower et al. The mobile sensing platform: An embedded activity recognition system. IEEE Pervasive Computing 7, 2: 32-41

15. Conrad Wall 3rd. 2010. Application of vibrotactile feedback of body motion to improve rehabilitation in individuals with imbalance. Journal of neurologic physical therapy: JNPT 34, 2: 98.

16. Barbara Datz-Kauffold and Shawn Lawton Henry. 2000. Waving magic wands: Interaction techniques to improve usability testing low-fidelity prototypes. Usability Professionals' Association, Asheville, NC.

17. Geoffrey B Duggan, Edmund Keogh, Gail A Mountain, Paul McCullagh, Jason Leake, and Christopher Eccleston. 2015. Qualitative evaluation of the SMART2 self-management system for people in chronic pain. Disability and rehabilitation. Assistive technology 10, 1: 53-60.

18. Sergio Felipe, Aneesha Singh, Caroline Bradley, Amanda Williams, and Nadia Berthouze. Roles for Personal Informatics in Chronic Pain. Proceedings of the 9th International Conference on Pervasive Computing Technologies for Healthcare. 161-168.

19. Robert J Gatchel, Yuan Bo Peng, Madelon L Peters, Perry N Fuchs, and Dennis C Turk. 2007. The biopsychosocial approach to chronic pain: scientific advances and future directions. Psychological bulletin 133, 4: 581-624.

20. Claude Ghez, R Luke Dubois, Thanassis Rikakis, and Perry R Cook. 2000. An auditory display system for aiding interjoint coordination. Proceedings of the 6th International Conference on Auditory Display (ICAD'00)

21. Diane Gromala, C Shaw, and M Song. 2009. Chronic pain and the modulation of self in immersive virtual reality. AAAI Fall Symposium 2009.

22. Erik Grönvall and Nervo Verdezoto. 2013. Beyond self-monitoring: understanding non-functional aspects of home-based healthcare technology. Proceedings of the ACM international joint conference on Pervasive and ubiquitous computing. 587-596 
23. Vicki Harding and Paul J Watson. 2000. Increasing Activity and Improving Function in Chronic Pain Management. Physiotherapy 86, 12: 619-630

24. Monika I Hasenbring and Jeanine A Verbunt. 2010. Fear-avoidance and endurance-related responses to pain: new models of behavior and their consequences for clinical practice. The Clinical journal of pain 26, 9: 747-753.

25. Eve Hoggan, Stephen A Brewster, and Jody Johnston. 2008. Investigating the effectiveness of tactile feedback for mobile touchscreens. Proceedings of the SIGCHI conference on Human factors in computing systems, pp. 1573-1582. ACM, 2008.

26. Andrew Hunt and Sandra Pauletto. 2006. The sonification of EMG data. Proceedings of the International Conference on Auditory Displays

27. Masaki Iguchi, Masaki Matsubara, Hideki Kadone, and Hiroko Terasawa. 2013. How is auditory EMG biofeedback effective for blind people? Proceedings of the International Conference on Auditory Displays, 307-310.

28. iPosture. Retrieved September 21, 2016 from http://www.iposture.com

29. Stephanie M Jansen-Kosterink, Rianne M H A Huis in t Veld, Christian Schönauer, et al. 2013. A Serious Exergame for Patients Suffering from Chronic Musculoskeletal Back and Neck Pain: A Pilot Study. Games for Health Journal 2, 5: 299-307.

30. Ru-Rong Ji, Tatsuro Kohno, Kimberly A Moore, and Clifford J Woolf. 2003. Central sensitization and LTP: do pain and memory share similar mechanisms? Trends in neurosciences 26.12. 696-705.

31. Rose Johnson, Nadia Bianchi-Berthouze, Yvonne Rogers, and Janet van der Linden. 2013. Embracing calibration in body sensing: using self-tweaking to enhance ownership and performance. Proceedings of the ACM international joint conference on Pervasive and ubiquitous computing. 811-820.

32. Evangelos Karapanos 2013. User experience over time. Modeling Users' Experiences with Interactive Systems. Springer Berlin Heidelberg, 57-83.

33. Agnes W K Lam, Danniel Varona-Marin, Yeti Li, Mitchell Fergenbaum, and Dana Kulić. 2015. Automated Rehabilitation System: Movement Measurement and Feedback for Patients and Physiotherapists in the Rehabilitation Clinic. HumanComputer Interaction 31, 3-4: 293-334.

34. A S Lee, J Cholewicki, N P Reeves, and B T Zazulak. 2010. Comparison of Trunk Proprioception Between Patients With Low Back Pain and Healthy Controls. Arch. physical medicine and rehabilitation 91.91327
35. Lumo Lift. Lumo Lift - Posture Coach \& Activity Tracker. Retrieved September 21, 2016 from http://www.lumobodytech.com/lumo-lift

36. Temitayo A. Olugbade., Nadia Bianchi-Berthouze, Nicolai Marquardt, and Amanda Williams. 2015. Pain level recognition using kinematics and muscle activity for physical rehabilitation in chronic pain. Proceedings of International Conference on Affective Computing \& Intelligent Interaction

37. James Rainville, Rob JEM Smeets, Tom Bendix, and Torill H Tveito. 2011. Fear-avoidance beliefs and pain avoidance in low back pain — translating research into clinical practice. The Spine Journal.

38. Giulio Rosati, Antonio Rodà, Federico Avanzini, and Stefano Masiero. 2013. On the Role of Auditory Feedback in Robot-Assisted Movement Training after Stroke: Review of the Literature. Computational Intelligence and Neuroscience. 3-4: 1-15.

39. Robert J Sanchez, Jiayin Liu, Sandhya Rao, et al. 2006. Automating Arm Movement Training Following Severe Stroke: Functional Exercises With Quantitative Feedback in a Gravity-Reduced Environment. IEEE Transactions on Neural Systems and Rehabilitation Engineering, 14, 3: 378-389.

40. Christian Schönauer, Thomas Pintaric, H Kaufmann, Stephanie Jansen Kosterink, and Miriam VollenbroekHutten. 2011. Chronic Pain Rehabilitation with a Serious Game using Multimodal Input. IEEE ICVR, 18 .

41. Aneesha Singh, Annina Klapper, Jining Jia, Antonio Fidalgo, Ana Tajadura-Jimenez, Amanda Williams, Nadia Bianchi-Berthouze 2014. Motivating people with chronic pain to do physical activity: opportunities for technology design. Proceedings of the 32nd annual ACM conference on Human factors in computing systems. 2803-2812

42. Aneesha Singh, Stefano Piana, Davide Pollarolo, Gualtiero Volpe, Giovanna Varni, Ana TajaduraJimenez, Amanda Williams, Antonio Camurri, Nadia Bianchi-Berthouze. 2016. Go-with-the-Flow: Tracking, Analysis and Sonification of Movement and Breathing to Build Confidence in Activity Despite Chronic Pain. Human-Computer Interaction 31, 3-4: 335-383.

43. Janet van der Linden, Erwin Schoonderwaldt, Jon Bird, and Rose Johnson. 2011. MusicJacket-Combining Motion Capture and Vibrotactile Feedback to Teach Violin Bowing. IEEE Transactions on Instrumentation and Measurement 60, 1: 104-113.

44. Johan W Vlaeyen and Steven J Linton. 2000. Fearavoidance and its consequences in chronic musculoskeletal pain: a state of the art. Pain 85, 3: 317-332. 
45. Katharina Vogt, David Pirrò, Ingo Kobenz, Robert Höldrich, and Gerhard Eckel. 2009. PhysioSonic evaluated movement sonification as auditory feedback in physiotherapy. Auditory display. Springer-Verlag 103-120

46. Raimundo della Volpe, T Popa, F Ginanneschi, R Spidalieri, R Mazzocchio, and A Rossi. 2006. Changes in coordination of postural control during dynamic stance in chronic low back pain patients. Gait \& Posture 24, 3: 349-355.

47. Mathias Wellner, Audrey Schaufelberger, and Robert Riener. 2007. A Study on Sound Feedback in a Virtual Environment for Gait Rehabilitation. IEEE Virtual Rehabilitation, 53-56.
48. Ni Zhu, Tom Diethe, Massimo Camplani, et al. Bridging e-Health and the Internet of Things: The SPHERE Project. IEEE Intelligent Systems 30, 4: 3946.

49. John Zimmerman, Jodi Forlizzi, and Shelley Evenson. 2007. Research through design as a method for interaction design research in HCI. Proceedings of the SIGCHI conference on Human factors in computing systems. 493-502. 